\title{
Critical exponents of plane meanders
}

\author{
Iwan Jensen* and Anthony J. Guttmann†, \\ Department of Mathematics \& Statistics, \\ The University of Melbourne, \\ Victoria 3010, Australia
}

November 5, 2018

\begin{abstract}
Meanders form a set of combinatorial problems concerned with the enumeration of self-avoiding loops crossing a line through a given number of points, $n$. Meanders are considered distinct up to any smooth deformation leaving the line fixed. We use a recently developed algorithm, based on transfer matrix methods, to enumerate plane meanders. This allows us to calculate the number of closed meanders up to $n=48$, the number of open meanders up to $n=43$, and the number of semi-meanders up to $n=45$. The analysis of the series yields accurate estimates of both the critical point and critical exponent, and shows that a recent conjecture for the exact value of the semi-meander critical exponent is unlikely to be correct, while the conjectured exponent value for closed and open meanders is not inconsistent with the results from the analysis.
\end{abstract}

Meanders form a set of unsolved combinatorial problems concerned with the enumeration of self-avoiding loops crossing a line through a given number of points [1]. Meanders are considered distinct up to any smooth deformation leaving the line fixed. This problem seems to date back at least to the work of Poincaré on differential geometry [2]. Since then it has from time to time been studied by mathematicians in various contexts such as the folding of a strip of stamps [3, 4] or folding of maps [0]. More recently it has been related to enumerations of ovals in planar algebraic curves [6] and the classification of 3-manifolds [7]. During the last decade or so it has received considerable attention in other areas of science. In computer science meanders are related to the sorting of Jordan sequences [8] and have been used for lower bound arguments [9]. In physics meanders are relevant to the study of compact foldings of polymers [10, 11], properties of the Temperley-Lieb algebra [12, 13], matrix models [14, 15], and models of low-dimensional gravity [16].

A closed meander of order $n$ is a closed self-avoiding loop crossing an infinite line $2 n$ times (see figure 1). The meandric number $M_{n}$ is simply the number of such meanders distinct up to smooth transformations. Note that each meander forms a single connected loop. The number of closed meanders is expected to grow exponentially, with a sub-dominant term given by a critical exponent, $M_{n} \sim C R^{2 n} / n^{\alpha}$. The exponential growth constant $R$ is often called the connective constant. Thus the generating function is expected to behave as

*e-mail: I.Jensen@ms.unimelb.edu.au

$\dagger$ †-mail: tonyg@ms.unimelb.edu.au 


$$
\mathcal{M}(x)=\sum_{n=1}^{\infty} M_{n} x^{n} \sim A(x)\left(1-R^{2} x\right)^{\alpha-1},
$$

and hence have a singularity at $x_{c}=1 / R^{2}$ with exponent $\alpha-1$. The first meandric numbers are $M_{1}=1, M_{2}=2$ and $M_{3}=8$. One can extend the definition to multi-component systems of closed meanders, where we allow configurations with several disconnected closed loops. The meandric numbers $M_{n}^{(k)}$ are then the number of meanders with $2 n$ crossings and $k$ independent loops.
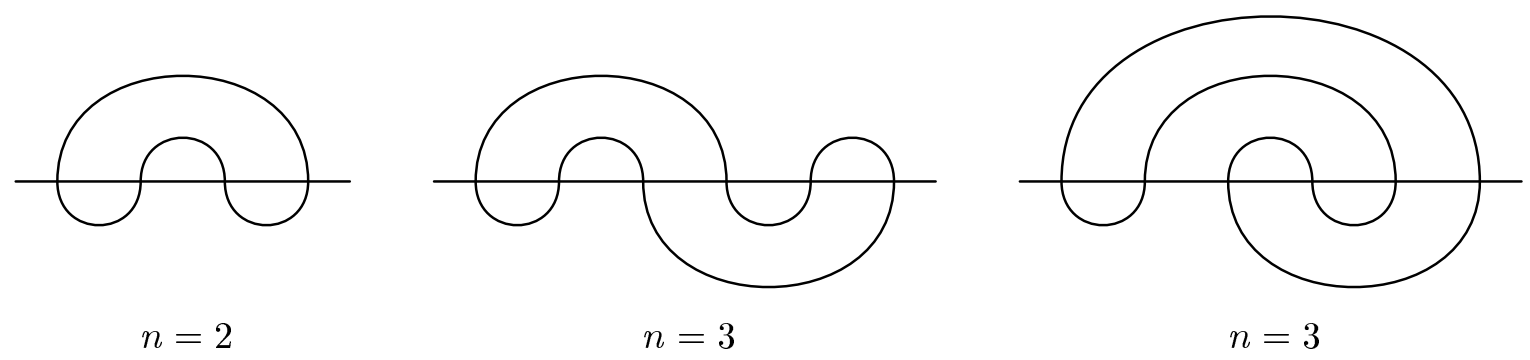

Figure 1: A few examples of closed meanders of order 2 and 3, respectively.

An open meander of order $n$ is a self-avoiding curve running from west to east while crossing an infinite line $n$ times. The number of such curves is $m_{n}$ and we can define a generating function for this problem in analogy with (1). It should be noted [1] that $M_{n}=m_{2 n-1}$, and hence the critical exponent is identical to that of closed meanders and the connective constant is $R$.

Finally, instead of looking at intersections with an infinite line one could consider a semiinfinite line and allow the curve to wind around the end-point of the line [10]. A semi-meander of order $n$ is a closed self-avoiding loop crossing the semi-infinite line $n$ times. The number of semi-meanders of order $n$ is denoted by $\bar{M}_{n} \sim C^{\prime} \bar{R} / n^{\bar{\alpha}}$ and we define a generating function as in (11). In this case a further interesting generalization is to study the number of semi-meanders $\bar{M}_{n}(w)$ which wind around the end-point of the line exactly $w$ times. Again we could also study systems of multi-component semi-meanders according to the number of independent loops. Two semi-meanders are shown in figure 2 .
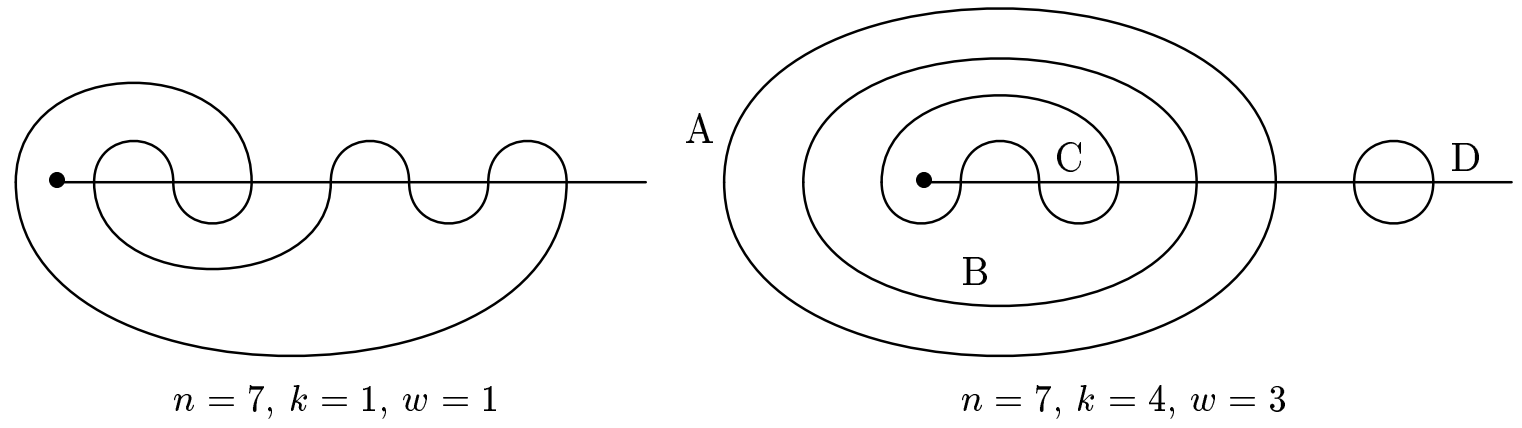

Figure 2: Two examples of semi-meanders. The first one has a single loop, wind around the origin once, and contain 7 crossings. The second semi-meander has 4 loops (labelled A-D), wind around the origin 3 times, and again contain 7 crossings.

In a recent paper it was argued that the meander problem is related to the gravitational version of a certain loop model [16]. From the conformal field theory of the model, conjectures were 
Table 1: The number, $M_{n}$, of connected closed meanders with $2 n$ crossings.

\begin{tabular}{rrrrrr}
\hline \hline$n$ & \multicolumn{1}{c}{$M_{n}$} & $n$ & \multicolumn{1}{c}{$M_{n}$} & $n$ & \multicolumn{1}{c}{$M_{n}$} \\
\hline 1 & 1 & 9 & 933458 & 17 & 59923200729046 \\
2 & 2 & 10 & 8152860 & 18 & 608188709574124 \\
3 & 8 & 11 & 73424650 & 19 & 6234277838531806 \\
4 & 42 & 12 & 678390116 & 20 & 64477712119584604 \\
5 & 262 & 13 & 6405031050 & 21 & 672265814872772972 \\
6 & 1828 & 14 & 61606881612 & 22 & 7060941974458061392 \\
7 & 13820 & 15 & 602188541928 & 23 & 74661728661167809752 \\
8 & 110954 & 16 & 5969806669034 & 24 & 794337831754564188184 \\
\hline \hline
\end{tabular}

proposed for the exact critical exponent of closed and open meanders, $\alpha=(29+\sqrt{145}) / 12=$ $3.4201328 \ldots$, as well as the exponent for semi-meanders, $\bar{\alpha}=1+\sqrt{11}(\sqrt{29}+\sqrt{5}) / 24=$ $2.0531987 \ldots$. This work has recently been extended to multi-component systems of closed and semi-meanders [17]. Conjectures were then given for the critical exponents as functions of the loop-fugacity $q$. These were checked numerically [17] and found to be correct within numerical error. In this Letter we analyse extended series for the meander generating functions. Using differential approximants we obtain accurate estimates for the exponents and find that the conjecture for the semi-meander exponent is unlikely to be correct, while the conjecture for closed meanders is not inconsistent with the results from the analysis.

The difficulty in the enumeration of most interesting combinatorial problems is that, computationally, they are of exponential complexity. Initial efforts at computer enumeration of meanders were based on direct counting. Lando and Zvonkin [1] studied closed meanders, open meanders and multi-component systems of closed meanders, while Di Francesco et al. "11 studied semi-meanders. In this Letter we use a new and improved algorithm [18, based on transfer matrix methods, to enumerate various meander problems such as closed, open and semi-meanders. The method is similar to the transfer matrix technique devised by Enting [19] in his pioneering work on the enumeration of self-avoiding polygons. The first terms in the series for the meander generating function can be calculated using transfer matrix techniques. This involves drawing an intersection perpendicular to the infinite line. Meanders are enumerated by successive moves of the intersection, so that one crossing at a time is added to the meanders. A preliminary description of the algorithm can be found in [18] and further details will appear elsewhere. A very closely related algorithm was used and described in [17].

The enumerations undertaken thus far are too numerous to detail here. We only give the results for connected closed meanders $M_{n}$, open meanders $m_{n}$, and semi-meanders which wind around the origin any number of times and have only a single loop $\bar{M}_{n}$. The numbers of such meanders are listed in Table 1 1 Table 3.

We analyzed the series by the numerical method of differential approximants [20]. Estimates of the critical point and critical exponents were obtained by averaging values obtained from inhomogeneous differential approximants chosen such that most, if not all, series terms were used. Some approximants were excluded from the averages because the estimates were obviously spurious. The error quoted for these estimates reflects the spread (basically one standard deviation) among the approximants. Note that these error bounds should not be viewed as a measure of the true error as they cannot include possible systematic sources of error. In Table 1 
Table 2: The number, $m_{n}$, of connected open meanders with $n$ crossings.

\begin{tabular}{rrrrrr}
\hline \hline$n$ & \multicolumn{1}{c}{$m_{n}$} & $n$ & \multicolumn{1}{c}{$m_{n}$} & $n$ & \multicolumn{1}{c}{$m_{n}$} \\
\hline 1 & 1 & 16 & 252939 & 31 & 5969806669034 \\
2 & 1 & 17 & 933458 & 32 & 15012865733351 \\
3 & 2 & 18 & 2172830 & 33 & 59923200729046 \\
4 & 3 & 19 & 8152860 & 34 & 151622652413194 \\
5 & 8 & 20 & 19304190 & 35 & 608188709574124 \\
6 & 14 & 21 & 73424650 & 36 & 1547365078534578 \\
7 & 42 & 22 & 176343390 & 37 & 6234277838531806 \\
8 & 81 & 23 & 678390116 & 38 & 15939972379349178 \\
9 & 262 & 24 & 1649008456 & 39 & 64477712119584604 \\
10 & 538 & 25 & 6405031050 & 40 & 165597452660771610 \\
11 & 1828 & 26 & 15730575554 & 41 & 672265814872772972 \\
12 & 3926 & 27 & 61606881612 & 42 & 1733609081727968492 \\
13 & 13820 & 28 & 152663683494 & 43 & 7060941974458059344 \\
14 & 30694 & 29 & 602188541928 & & \\
15 & 110954 & 30 & 1503962954930 & & \\
\hline \hline
\end{tabular}

Table 3: The number, $\bar{M}_{n}$, of connected semi-meanders with $n$ crossings.

\begin{tabular}{rrrrrr}
\hline \hline$n$ & \multicolumn{1}{c}{$\bar{M}_{n}$} & $n$ & \multicolumn{1}{c}{$\bar{M}_{n}$} & $n$ & \multicolumn{1}{c}{$\bar{M}_{n}$} \\
\hline 1 & 1 & 16 & 1053874 & 31 & 42126805350798 \\
2 & 1 & 17 & 3328188 & 32 & 137494070309894 \\
3 & 2 & 18 & 10274466 & 33 & 455792943581400 \\
4 & 4 & 19 & 32786630 & 34 & 1493892615824866 \\
5 & 10 & 20 & 102511418 & 35 & 4967158911871358 \\
6 & 24 & 21 & 329903058 & 36 & 16341143303881194 \\
7 & 66 & 22 & 1042277722 & 37 & 54480174340453578 \\
8 & 174 & 23 & 3377919260 & 38 & 179830726231355326 \\
9 & 504 & 24 & 10765024432 & 39 & 600994488311709056 \\
10 & 1406 & 25 & 35095839848 & 40 & 1989761816656666392 \\
11 & 4210 & 26 & 112670468128 & 41 & 6664356253639465480 \\
12 & 12198 & 27 & 369192702554 & 42 & 22124273546267785420 \\
13 & 37378 & 28 & 1192724674590 & 43 & 74248957195109578520 \\
14 & 111278 & 29 & 3925446804750 & 44 & 247100408917982623532 \\
15 & 346846 & 30 & 12750985286162 & 45 & 830776205506531894760 \\
\hline \hline
\end{tabular}


Table 4: Estimates of the critical points and exponents of the meander generating functions for closed, open and semi-meanders, as obtained from 2. order differential approximants. $L$ is the degree or the inhomogeneous polynomial.

\begin{tabular}{rlccccl}
\hline \hline & \multicolumn{2}{c}{ Closed meanders } & \multicolumn{2}{c}{ Open meanders } & \multicolumn{2}{c}{ Semi-meanders } \\
\hline $\mathrm{L}$ & \multicolumn{1}{c}{$x_{c}$} & $\alpha-1$ & \multicolumn{1}{c}{$x_{c}$} & $\alpha-1$ & $x_{c}$ & $\bar{\alpha}-1$ \\
\hline 0 & $0.08154671(24)$ & $2.42104(42)$ & $0.28556361(40)$ & $2.42129(36)$ & $0.285564437(10)$ & $1.053693(12)$ \\
1 & $0.08154684(14)$ & $2.42084(33)$ & $0.28556416(19)$ & $2.42075(20)$ & $0.28556448(10)$ & $1.05362(16)$ \\
2 & $0.081546912(59)$ & $2.42079(45)$ & $0.28556418(64)$ & $2.42072(63)$ & $0.28556447(13)$ & $1.05358(27)$ \\
3 & $0.081546916(84)$ & $2.42069(17)$ & $0.28556386(33)$ & $2.42109(34)$ & $0.285564436(31)$ & $1.053693(47)$ \\
4 & $0.081546950(46)$ & $2.42061(10)$ & $0.28556390(50)$ & $2.42101(46)$ & $0.285564433(29)$ & $1.053700(34)$ \\
5 & $0.081546901(82)$ & $2.42074(18)$ & $0.28556406(10)$ & $2.42088(13)$ & $0.285564437(24)$ & $1.053692(32)$ \\
6 & $0.081546917(67)$ & $2.42065(21)$ & $0.28556394(28)$ & $2.42101(32)$ & $0.285564425(65)$ & $1.053699(96)$ \\
7 & $0.081546910(72)$ & $2.42070(21)$ & $0.28556407(10)$ & $2.42088(12)$ & $0.285564413(58)$ & $1.053717(71)$ \\
8 & $0.08154682(16)$ & $2.42090(30)$ & $0.28556408(10)$ & $2.42087(13)$ & $0.285564425(46)$ & $1.053706(54)$ \\
9 & $0.08154668(32)$ & $2.42115(60)$ & $0.285564096(83)$ & $2.42083(11)$ & $0.285564434(52)$ & $1.053692(79)$ \\
10 & $0.08154671(26)$ & $2.42107(45)$ & $0.28556414(16)$ & $2.42078(18)$ & $0.285564433(47)$ & $1.053695(63)$ \\
\hline \hline
\end{tabular}

we have listed the results from this analysis. Our first observation is that the critical points of open and semi-meanders are identical, and thus so are the connective constants $R$ for all the problems (recall that for closed meanders $x_{c}=1 / R^{2}$ ). Clearly the most accurate estimates are obtained from the semi-meander series and from this we estimate, conservatively, that $x_{c}=0.2855644(2)$ and thus $R=3.501837(3)$. Secondly, as expected open and closed meanders have the same critical exponent, which we estimate to be $\alpha=3.4208(6)$. This could, though only marginally, be consistent with the conjectured value $\alpha=(29+\sqrt{145}) / 12=3.4201328 \ldots$. For semi-meanders we estimate $\bar{\alpha}=2.0537(2)$, which is not consistent with the conjecture $\bar{\alpha}=1+\sqrt{11}(\sqrt{29}+\sqrt{5}) / 24=2.0531987 \ldots$

In order to gain a better understanding of the behaviour of the exponent estimates it is useful to plot them against the number of terms used to form the differential approximant. In particular we can check whether or not the estimates asymptote or whether they are drifting with the length of the series. In figure 3 we have done this for semi-meanders and open meanders. These plots strongly reinforce the comments made above. The exponent estimates for semimeanders increase as more terms are used and appear to settle down to an asymptotic value above the conjectured value. For open meanders the exponent estimates decrease and approach the conjectured value as more terms are used. It is quite likely that with a longer series the estimates would actually converge to the conjectured value, though it is also possible that the estimates could settle at a value just above the conjectured value. If we look at the estimates in Table 4 we note that for both open and closed meanders the exponent estimates decrease as the critical point estimates increase. It is possible that as $x_{c}$ approaches the estimate obtained from semi-meanders the exponent estimates approach the conjectured value. To check this we plotted (in figure 4) the exponent estimates vs the critical point estimates for open and closed meanders. The solid lines are the conjectured exponent value and the best estimate for the critical point based on the semi-meander analysis. Clearly the estimates pass extremely close to the intersection between the solid lines, lending further support to the possibility that the conjecture for $\alpha$ is correct.

Next we looked for non-physical singularities and found that both the open and semi- 

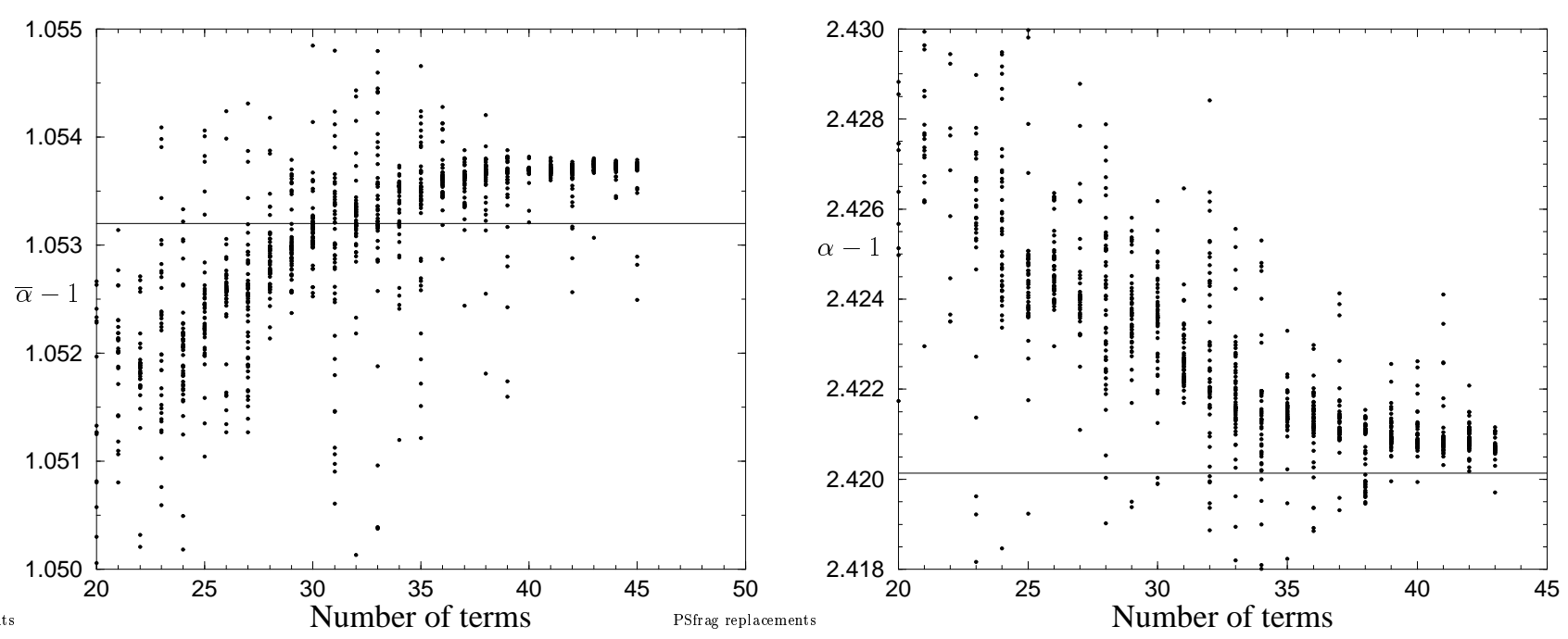

Figure 3: Estimates of the critical exponents of the semi-meander generating function, $\bar{\alpha}-1$, and the open meander generating function, $\alpha-1$, vs. the number of terms from the series used by the differential approximants. Each point represents an estimate obtained from a particular 2. order differential approximant.
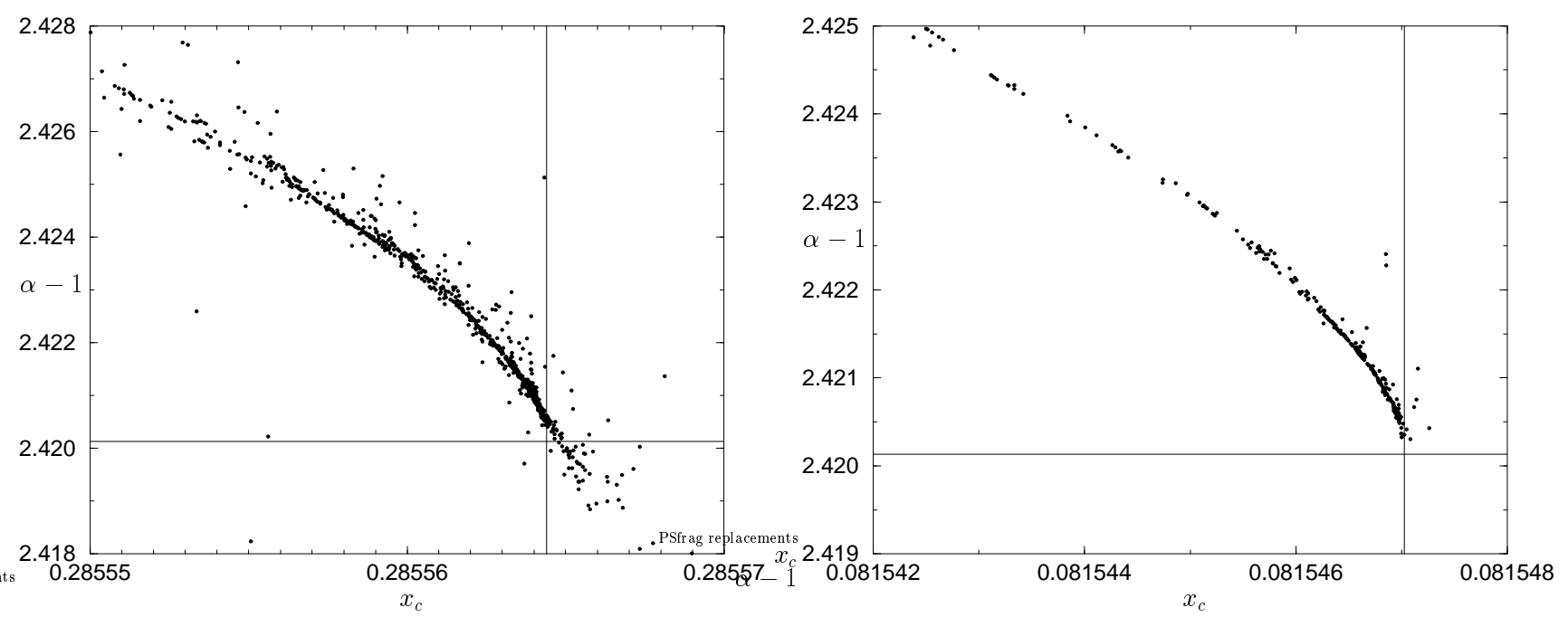

Figure 4: Estimates of the critical exponents for open (left panel) and closed meanders vs. the corresponding critical point estimates. 
meander generating functions have a singularity at $-1 / R$ with an exponent whose value is consistent with $\alpha-1$. These generating functions also have a pair of singularities in the complex plane at $\pm 0.685(5) i$. The exponent estimates are quite poor, but consistent with the value $\alpha-1$.

Finally we turned our attention to the "fine-structure" of the asymptotic behaviour of the meandric numbers,

$$
\begin{aligned}
M_{n} & \sim R^{2 n} \sum_{i=0} c_{i} / n^{\alpha+f(i)}, \\
m_{n} & \sim R^{n} \sum_{i=0}\left[c_{i} / n^{\alpha+f(i)}+(-1)^{n} d_{i} / n^{\alpha+f(i)}\right] \\
\bar{M}_{n} & \sim R^{n} \sum_{i=0}\left[c_{i} / n^{\bar{\alpha}+f(i)}+(-1)^{n} d_{i} / n^{\alpha+f(i)}\right] .
\end{aligned}
$$

The alternating sign terms are due to the singularity at $-1 / R$. Fitting the meandric numbers to these formulas we found excellent convergence when $f(i)=i$. This corresponds to the case where there are only analytic corrections-to-scaling terms. The leading amplitudes $c_{0}$ are of special interest, and for closed, open and semi-meanders we found the values $0.339(1), 11.45(3)$, and $0.688(1)$, respectively.

\section{E-mail or WWW retrieval of series}

The series for the various generating functions studied in this paper can be obtained via e-mail by sending a request to I.Jensen@ms.unimelb.edu.au or via the world wide web on the URL http://www.ms.unimelb.edu.au/ iwan// by following the instructions.

\section{Acknowledgements}

We would like to thank Di Francesco et al. for sending us their papers prior to publication. Financial support from the Australian Research Council is gratefully acknowledged.

\section{References}

[1] S. K. Lando and A. K. Zvonkin, Theoret. Comput. Sci. 117, 227 (1993).

[2] H. Poincaré, Rend. Circ. Mat. Palermo 33, 375 (1912).

[3] J. Touchard, Canad. J. Math. 2, 385 (1950).

[4] J. E. Koehler, J. Combin. Theory 5, 135 (1968).

[5] W. Lunnon, Math. Comp. 22, 193 (1968).

[6] V. Arnold, Siberian Math. J., 29717 (1988).

[7] K. H. Ko and L. Smolinsky, Pacific J. Math. 149, 319 (1991). 
[8] K. Hoffmann, K. Mehlhorn, P. Rosenstiehl, and R. E. Tarjan, Information and Control 68, 170 (1988).

[9] N. Alon and W. Maass, J. Comput. System Sci. 37, 118 (1988).

[10] P. Di Francesco, O. Golinelli and E. Guitter, Math. Comput. Modelling 26, 97 (1997).

[11] P. Di Francesco, O. Golinelli and E. Guitter, Nucl. Phys. B 482, 497 (1996).

[12] P. Di Francesco, O. Golinelli and E. Guitter, Commun. Math. Phys. 186, 1 (1997).

[13] P. Di Francesco, Commun. Math. Phys. 191, 543 (1998); J. Math. Phys. 38, 5905 (1997).

[14] Y. Makeenko, Nucl. Phys. Proc. Suppl. 49, 226 (1996).

[15] G. W. Semenoff and R. J. Szabo, Int. J. Mod. Phys. A12, 2135 (1997).

[16] P. Di Francesco, O. Golinelli and E. Guitter, Nucl. Phys. B 570, 699 (2000).

[17] P. Di Francesco, E. Guitter and J. L. Jacobsen, preprint http:://arxiv.org/abs/condmat/0003008.

[18] I. Jensen, preprint http:://arxiv.org/abs/cond-mat/9910313

[19] I. G. Enting, J. Phys. A 13, 3713 (1980).

[20] A. J. Guttmann, in Phase Transitions and Critical Phenomena, Vol. 13, eds. C. Domb and J. L. Lebowitz, Academic Press, New York (1989), pp 1-234. 